der einer Keimverdünnungsreihe in vitro entspricht. Eine mehrfache Spülung ist also effektiv und wird durch Wiederholung bis zu fünfmal zu dem gewünschten Erfolg einer beträchtlichen Keimverringerung führen, bis zu einem Bereich, der von den Tieren toleriert wird.

Die von uns durchgeführten Versuche zeigten bei den Tieren eine deutliche Verlängerung der ťberlebenszeit nach intraabdominaler Spülung. Die Versuche mit Dauerspülung waren dagegen ohne Erfolg und brachten eine Reihe zusätzlicher Komplikationen.

Auf Grund unserer Ergebnisse empfehlen wir eine intraoperative mehrmalige ausgiebige Spülung (Verminderung der Keimzahl), Instillation von Breitbandantibiotica und i.v. Gabe von Antibiotica nach Resistenzbestimmung der nach Abstrich gewonnenen Keime.

Präsident: Sie sehen aus den folgenden Themen, wie wichtig offenbar doch die subphrenischen, subhepatischen Abscesse und transdiaphragmatischen Fisteln sind; $d . h$. welche Schwierigkeiten sie den Kollegen bereiten.

Das Manuskript der Zusammenfassung zu dem Vortrag 308, Diagnostik und Therapie der subphrenischen, subhepatischen Abscesse und transdiaphragmatischen Fisteln von H. G. Kühn-Berlin, wurde nicht vorgelegt.

Präsident: Eine sehr interessante Beobachtung. Herr Kollege Hess aus Zürich, den ich gern über die postoperative Cholorrhoe bei biliärer Cirrhose hätte sprechen lassen, ist leider erkrankt und hat mir das telegraphisch mitgeteilt. Er kann heute nicht sprechen.

\title{
309. Die postoperative Cholorrhoe
}

\section{W. Hess-Zürich/Schweiz \\ Postoperative Cholorrhoea}

Summary. In cases of severe biliary obstruction as well as of alcoholic hepatic cirrhosis an abnormally high bile production, 2000 to $6000 \mathrm{ml}$, was found postoperatively. In the cases of biliary obstruction we are dealing with a secretinregulated secretion, inhibited by acetazolamide and rich in bicarbonates, from the duct system whose surface is greatly enlarged. In cirrhosis, on the other hand, a transsudate poor in bicarbonates is produced, apparently caused haemodynamically by postsinusoidal obstruction of the hepatic flow bed. In none of the cases was the production of bilirubin or bile acids increased. 5 observations are described.

Zusammenfassung. Sowohl bei Fällen mit schwerer Gallenwegsobstruktion wie bei alkoholischen Lebercirrhosen wurde eine abnorm hohe Gallenproduktion, 2000-6000 ml, postoperativ festgestellt. Bei den Fällen mit Gallenwegsobstruktion handelt es sich um eine durch Sekretin gesteuerte, mit Acetazolamid hemmbare, bicarbonatreiche Sekretion aus dem Gangsystem, dessen Oberfläche stark vergrößert ist. Bei Cirrhosen dagegen wird ein bicarbonatarmes Transsudat produziert, das, wie die vermehrte Lymphproduktion bei Cirrhosen, offenbar hämodynamisch durch die postsinusoidale Obstruktion des Leberstrombettes zustandekommt. In keinem Falle war der Ausstoß an Bilirubin oder Gallensäuren vermehrt. Fünf einschlägige Beobachtungen werden beschrieben. 